\title{
Neutrophil alkaline phosphatase score in chronic granulocytic leukaemia: effects of splenectomy and antileukaemic drugs
}

\author{
A. S. D. SPIERS, A. LIEW, AND A. G. BAIKIE
}

From the Medical Research Council Leukaemia Unit, Royal Postgraduate Medical School, and Hammersmith Hospital, Ducane Road, London W12 OHS, and the University of Tasmania Department of Medicine, Royal Hobart Hospital, Hobart, Tasmania, Australia 7000

SYNOPSIS Staining with naphthol AS phosphate and Fast Blue BB salt has been used for the estimation of neutrophil alkaline phosphatase (NAP) scores in patients with chronic granulocytic leukaemia (CGL). The very low scores found at diagnosis rise when the disease is treated, and there is some inverse correlation between the NAP score and the absolute neutrophil count. Patients treated intensively developed high NAP scores. Elective splenectomy performed during the chronic phase of CGL is followed by a pronounced but transient neutrophilia and a concurrent striking rise in the NAP score. Similar changes were observed in patients without CGL who underwent splenectomy. These observations can be explained by assuming that newly formed neutrophils in CGL have a normal content of NAP but are rapidly sequestered in non-circulating extramedullary pools, whereas the circulating neutrophil with a typically low NAP content is a relatively aged cell which has lost enzyme activity. In subjects with or without CGL, removal of the spleen, a major site of such pooling, temporarily permits the circulation of newly formed neutrophils but eventually other organs assume the sequestering functions of the spleen. Thus the aberrations of NAP score seen in CGL might be attributable not to an intrinsic cellular defect but to an exaggeration of the granulocyte storage phenomena which also occur in subjects without CGL.

Almost 30 years ago, Gomori's histochemical method for the demonstration of alkaline phosphatase was applied to the localization of the enzyme in human peripheral blood and bonemarrow cells: it appeared to be confined to mature neutrophils and band forms (Wachstein, 1946). Wachstein described increased enzyme activity in polycythaemia rubra vera, some cases of myelosclerosis, and in leukaemoid reactions. He was also the first to report a pronounced decrease in activity in most cases of chronic granulocytic leukaemia (CGL), and this difference has been used as an aid in the differentiation of CGL from the above conditions (Koler, Seaman, Osgood, and Van Bellinghen, 1958; Mitus, Bergna, Mednicoff, and Dameshek, 1958). Reduced levels of the enzyme are also observed in paroxysmal nocturnal haemoglobinuria and infectious mononucleosis (Tanaka, Valentine, and Fredricks, 1960; Lewis and Dacie, 1965). The

Received for publication 29 January 1975. enzyme was termed leucocyte alkaline phosphatase (LAP), but its virtual restriction to neutrophil polymorphonuclear cells has led to increasing use of the term neutrophil alkaline phosphatase (NAP).

Increased NAP activity has been observed in myeloproliferative disorders, infections, after administration of adrenocortical steroids (Strausz, Kékes, and Szebeni, 1965), in carcinomatosis (Lille-Szyszkowicz, Gabay, Saracino, and Bourdin, 1966), and in Hodgkin's disease (Bennett, Nathanson, and Rutenburg, 1968; Sforza, De Somoza, and Morgenfeld, 1971; Simmons, Spiers, and Fayers, 1973). In CGL, a low NAP value is almost invariable in untreated patients (Rosner, Schreiber, and Parise, 1972), and in addition, the enzyme purified from CGL neutrophils is reported to have a lower specific activity than NAP from normal cells (Bottomley, Lovig, Holt, and Griffin, 1969): the mechanism of these changes is unknown. We have studied NAP activity in patients with CGL before and during treatment, and during this investi- 
gation found an unexpected and striking alteration of the NAP level following splenectomy.

\section{Patients and Methods}

\section{PATIENTS}

The 18 patients studied had haematologically typical CGL in its chronic phase, and in all the Philadelphia $\left(\mathrm{Ph}^{1}\right)$ chromosome was demonstrated in bone marrow cells. Fourteen of the 18 patients were untreated when first studied and 15 were studied during their initial chemotherapy. These 15 patients were splenectomized, and eight were studied within the 14 days after splenectomy. In 12 of the 15, the NAP was estimated six months after splenectomy. Thirteen patients were treated with busulphan while five received thioguanine for initial therapy as part of a clinical trial. The splenectomies were performed after the induction of disease remission, as part of a trial of elective splenectomy early in the course of CGL (Baikie, 1968; Spiers, 1973). Observations on NAP activity made in patients whose CGL had transformed from the chronic phase to an acute or subacute phase were not included in analyses for this report.

NEUTROPHIL ALKALINE PHOSPHATASE SCORES Blood films were freshly made without the use of anticoagulants and fixed for 30 seconds in formalin: methanol $(1: 9)$ at $4^{\circ} \mathrm{C}$. They were stained immediately or stored in the dry state at $-20^{\circ} \mathrm{C}$ : control films stored in the same way showed no detectable change in NAP score over several months. The fixed films were stained for NAP by the method of Rutenburg, Rosales, and Bennett (1965), using naphthol AS phosphate as the substrate and Fast Blue $\mathrm{BB}$ as the diazonium salt (both reagents from Sigma Chemical Company). We have found this method consistently successful and more satisfactory than the older method in which brentamine fast garnet is used. Nuclei were counterstained with $0.1 \%$ Neutral Red. The NAP activity was recorded as a weighted score, determined by examining 100 polymorphonuclear cells under oil immersion and grading each cell from 0 to 4 depending on the numbers of blue granules it contained. The intensity of coloration of the granules is disregarded and the final score is the sum of the 100 readings. The possible score therefore ranges from 0 to 400 and the normal range for our laboratory is 37 to 98 . All films were scored by one of us and many were scored independently by two. The range of betweenobserver variation was less than $5 \%$. The characteristics by which neutrophils were graded as 1, 2, 3, or 4 have been illustrated by Rutenburg and his colleagues (1965). This staining method is more

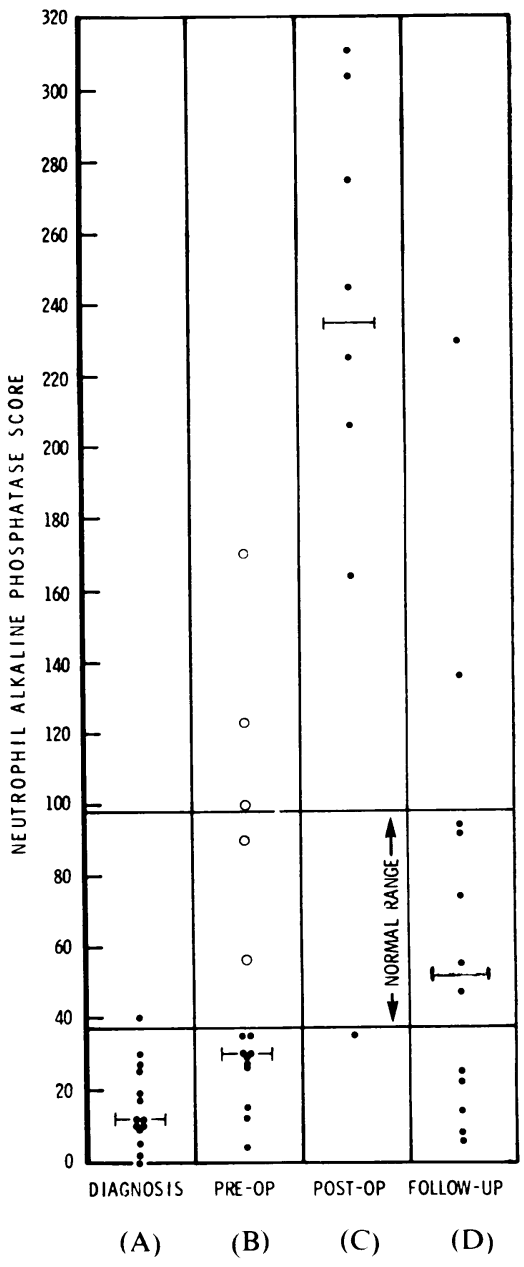

Fig 1 Neutrophil alkaline phosphatase (NAP) scores in patients with chronic granulocytic leukaemia (CGL). Observations made $(A)$ at diagnosis, when all patients had a leucocytosis and neutrophilia; $(B)$ immediately before splenectomy, when all patients had received antileukaemic drugs and had neutrophil counts in or close to the normal range; $(C)$ the peak NAP scores in individual patients during the first 14 postoperative days (postoperative observations were not made in some of the earliest splenectomies); and (D) NAP scores six months after splenectomy. The horizontal bars are the medians of each set of observations. The median value after treatment is higher than at diagnosis: the five patients represented by open circles had the highest scores and all had received relatively more intensive chemotherapy using thioguanine: the other patients were treated with busulphan. The marked rise in NAP score postoperatively $(C)$ is not sustained but even at six months $(D)$ the median score is higher than at any time before splenectomy. 
sensitive than those which it now supersedes, and scores of zero for 100 cells are very rare, even in untreated CGL in its chronic phase. The absolute neutrophil count was obtained from the percentage of segmented neutrophils in a differential count of 100 Romanowsky-stained white cells.

\section{Results}

NAP SCORES IN UNTREATED CGL

In 13 of the 14 untreated patients the NAP score was below the normal range (fig 1) and in one the score was 40 (mean for 14 patients 15.6).

\section{RELATION OF NAP SCORE TO NEUTROPHIL}

COUNT

There appeared to be some inverse correlation between NAP score and the absolute neutrophil count in the peripheral blood. Just before splenectomy, when patients had received chemotherapy for CGL and had neutrophil counts close to the normal range,

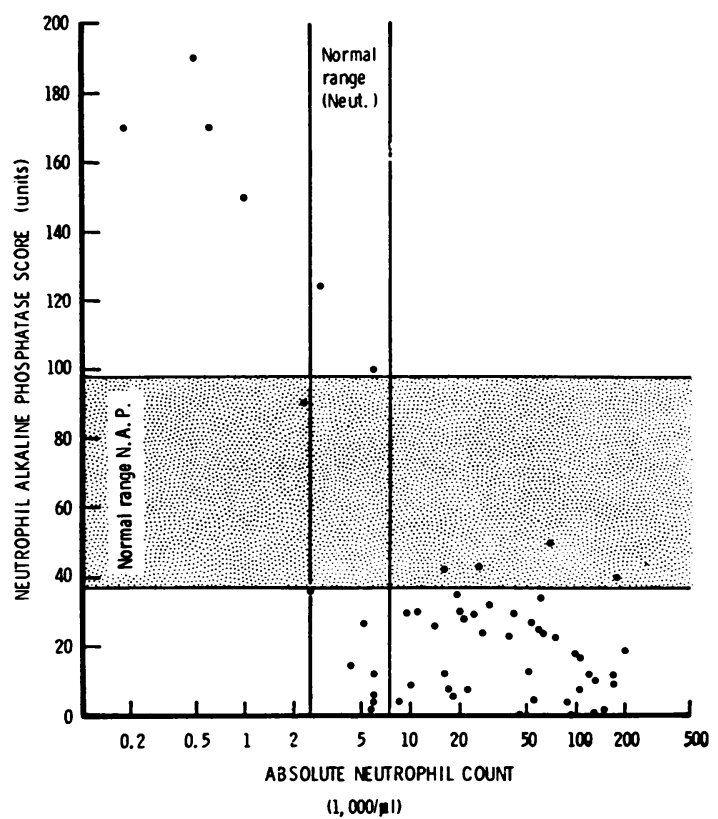

Fig 2 Effects of antileukaemic chemotherapy and the associated changes in absolute neutrophil count upon NAP scores. The 55 observations plotted were made at different times in 18 patients with haematologically typical Ph $^{1}$-positive CGL. All were in the chronic phase of their disease and none had undergone splenectomy. Whereas low NAP scores may be associated with normal or grossly elevated neutrophil counts, high scores are never associated with an increased count. No observation fell within the normal range for both parameters. the mean NAP score for 15 patients was 32.0 ; in two patients the NAP score was normal and in three it was above normal (fig 1). The five highest scores were observed in patients who had had intensive treatment with thioguanine, and the highest individual reading (170) was observed in the patient with the lowest neutrophil count $(200$ per $\mu \mathrm{l})$.

Figure 2 further illustrates the same correlation. Fifty-five observations were made in the 18 patients studied at diagnosis and/or before splenectomy was performed. Although low NAP scores were most often associated with an increased neutrophil count, they were also seen on six occasions when the neutrophil count was normal. On the other hand, normal or high NAP scores were never found with an increased absolute neutrophil count.

Figure 3 shows the changes in NAP score and absolute neutrophil count in a patient with CGL who was followed for almost two years. The NAP score remained above or within the normal range for nearly one year: consistently low NAP scores in the second year were accompanied by normal or elevated neutrophil counts, and the reciprocity of the two curves is readily apparent.

EFFECTS OF SPLENECTOMY UPON NAP SCORE Fifteen patients whose CGL was in its chronic phase underwent elective splenectomy but in the first

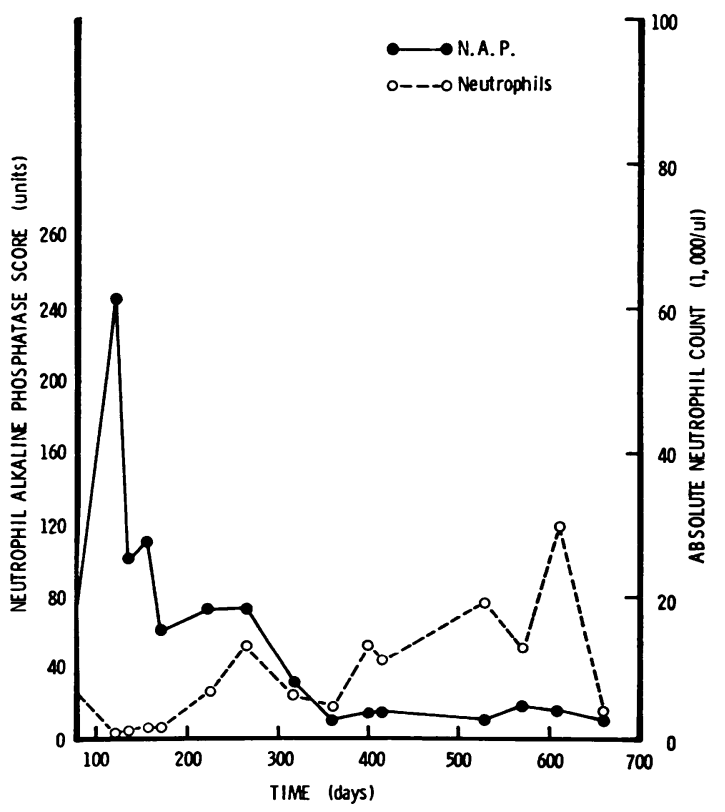

Fig 3 Serial neutrophil counts and NAP scores in a patient with CGL. The NAP scores became subnormal only when an absolute neutrophilia developed. 


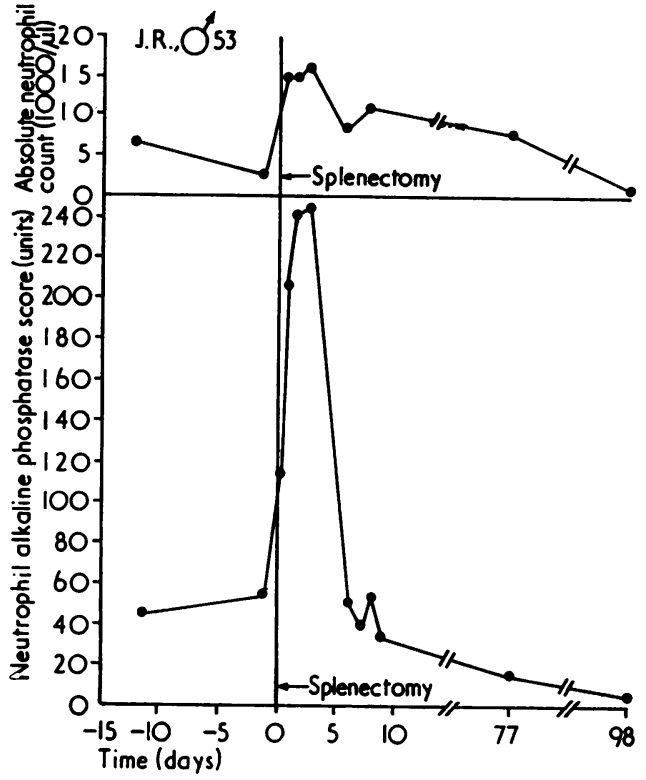

Fig 4 Immediate effects of splenectomy upon NAP score and absolute neutrophil count in a patient with CGL. No antileukaemic drugs were given from 34 days before operation until nine days afterward. A rapid sixfold increase in neutrophil count subsided spontaneously, while a similar rise in NAP score followed a virtually identical course. This direct correlation between neutrophil count and NAP score contrasts with the inverse correlation between these parameters seen when elevated neutrophil counts are due not to splenectomy but to uncontrolled CGL ( fig 2).

seven the NAP activity was not studied in the immediate postoperative period. In eight patients the NAP score was estimated repeatedly and in seven it became grossly elevated after splenectomy (fig 1); the mean for the eight patients was $221 \cdot 0$. Serial observations in one of the patients are shown in figure 4. The neutrophil count rose sixfold in the 18 hours after splenectomy and fell spontaneously before antileukaemic chemotherapy was reinstituted. The NAP score showed a sixfold increase with a similar spontaneous fall which temporally was almost exactly parallel to the spontaneously decreasing neutrophil count. Thereafter the NAP score declined further and by 98 days from operation it was subnormal. Similar curves were obtained for other patients who underwent splenectomy and are summarized in fig 1, where it is seen that the NAP scores six months after operation are lower than the immediate postoperative values, but the median and mean values ( 51.0 and 67.0 respectively) nevertheless remain higher than the corresponding values at diagnosis or immediately before splenectomy. In no case was a postoperative rise in NAP score attributable to wound or other infection.

\section{OTHER OBSERVATIONS}

(a) In CGL, a rise in the NAP score following splenectomy is not related to previous chemotherapy. One patient, not included in the figures, was treated exclusively by leucapheresis on an Aminco continuous-flow blood-cell separator before splenectomy. On one occasion the NAP score was estimated before and immediately after leucapheresis: the score was 30 before the procedure and 38 afterward. Although repeated leucaphereses led to $i$ regression of splenomegaly, no marked rise in NAP score occurred. Before splenectomy this patient's or NAP score was 12, and it rose to 153 on the first $\vec{v}$ postoperative day.

(b) Elevation of the NAP score after splenectomy is not peculiar to patients with CGL. A patient with $\subseteq$ myelosclerosis had a score of 14 before splenectomy and 110 afterward. In a man with an insulinoma, $\vec{\varphi}$ splenectomy was performed for ease of surgical or access at pancreatectomy: his NAP score was 130 two days before operation, 260 one day postoperatively, and 140 by the 13 th day after surgery. (c) A rising NAP score was common but not invariable when CGL underwent metamorphosis to $\mathbb{D}$ a refractory phase: the alteration in score was not $\stackrel{2}{\Rightarrow}$ sufficiently consistent to be of much value in $\frac{0}{3}$ establishing the diagnosis of metamorphosis in difficult cases. After frank metamorphosis the NAP score often fluctuated violently in relation to cytotoxic drug therapy, but an inverse correlation ? with the absolute neutrophil count, as seen in the chronic phase of CGL, was no longer apparent.

\section{Discussion}

The NAP score is a summation of the individual $D$ scores in 100 neutrophils, and in the presence of gross neutrophilia a low score is still compatible $N$ with the presence of a normal absolute number of neutrophils bearing NAP. In an untreated CGL 0 patient with 50000 neutrophils per $\mu l$ and a NAP $\omega$ score of 6, drug treatment which reduced the neutrophil count to 5000 per $\mu \mathrm{l}$ would raise the NAP score 0 to 60 if neutrophils without NAP activity were $\Phi$ eliminated selectively and their diluting effect upon $\stackrel{\oplus}{+}$ the score removed. In CGL, serial observations of $\frac{T}{T}$ NAP score during treatment suggest that this some- $\frac{O}{\mathbb{D}}$ times occurs. Rosner and his colleagues (1972) and $\stackrel{?}{P}$ other workers (Mitus et al, 1958; King, Gillis, and $\stackrel{\otimes}{\stackrel{Q}{2}}$ Baikie, 1962; Block, Carbone, Oppenheim, and $\bar{\sigma}$ Frei, 1963) have reported that NAP scores sometimes return to normal when CGL has been con-o 
trolled by therapy. This change is inconstant; thus Rosner's group found restoration of a normal NAP score in only four of 16 patients, and in these four the score was not normal in every period of remission which they experienced. In our patients, the mean and median NAP scores were higher after drug treatment than before it, but in 10 patients the score was still subnormal (fig 1). Our finding of a loose inverse correlation between absolute neutrophil count and NAP score (fig 2) accords well with the observations of others that successful treatment of CGL has an inconstant association with an increase in previously depressed NAP scores. In our patients, restoration of the NAP score was not associated with a greatly increased proportion of neutrophils with NAP activity graded as 3 or 4 -these remained uncommon-but rather with a decrease in the proportion of neutrophils with zero activity. It therefore appears that drug treatment which corrects the neutrophilia in CGL may selectively affect that part of the neutrophil population which lacks alkaline phosphatase.

Our finding in a series of patients of a consistent striking increase in NAP score immediately after splenectomy has not to our knowledge been reported previously. Stewart (1974) noted an elevated NAP score in a single patient with CGL studied five days after splenectomy. The rise in NAP score in our patients accompanied a marked rise in neutrophil count, which is the reverse situation to the smaller increase in NAP score sometimes seen when CGL is treated with busulphan. The rise in NAP score after splenectomy was, as with drug therapy, associated with a decrease in the proportion of NAP-negative neutrophils rather than an increase in the numbers of cells with scores of 3 or 4 units. This effect of splenectomy appears to be independent of drug therapy, since it was also observed in the patient treated by leucapheresis alone. Similar marked elevations of NAP score after splenectomy were observed in a patient with myelosclerosis and a patient with no haematological disorder, so the phenomenon is not peculiar to CGL. However, its occurrence in CGL suggests a possible mechanism for the fluctuations of NAP score in this disease.

The contradictory literature relating to the interpretation of NAP values in CGL was reviewed by Pedersen and Hayhoe (1971a). Hypotheses which relate the loss of enzyme activity directly to possession of the $\mathrm{Ph}^{1}$ chromosome (Alter, Dobkin, Pourfar, Rosner, and Lee, 1963) seem incompatible with the return of NAP activity in diffusion chamber cultures (Chikkappa, Boecker, Borner, Carsten, Conkling, Cook, Cronkite, and Dunwoody, 1973) and in vivo during remission of CGL while the bonemarrow cells remain $\mathrm{Ph}^{1}$-positive (Tough, Jacobs,
Court Brown, Baikie, and Williamson, 1963). Normal or increased NAP activity and persisting $\mathrm{Ph}^{1}$-positivity is also seen in some cases of CGL after acute transformation (King et al, 1962; Hammouda, Quaglino, and Hayhoe, 1964). Other hypotheses which sought to explain elevated NAP scores in Down's syndrome and decreased scores in CGL as dose-effects of the NAP gene(s) were early seen to be unsatisfactory (King et al, 1962) and later became untenable with the demonstration (O'Riordan, Robinson, Buckton, and Evans, 1971) that the $\mathrm{Ph}^{1}$ anomaly involves a chromosome different from that which is present in triplicate in Down's syndrome.

The interpretation advanced by Hook and Engel (1964) seems to us to fit best all the observations including those we now report for the first time. They suggested that low NAP scores in CGL were due to the accumulation of ageing neutrophils with reduced enzyme activity. Such cells diluted the younger neutrophils which possessed NAP activity and thereby lowered the NAP score, even though the absolute number of NAP-carrying neutrophils might be normal. This interpretation is compatible with the prolonged intravascular life-span of mature granulocytes in CGL (Galbraith, 1966). Recent studies by Kelemen (1973), and our own observations of the effects of splenectomy upon the NAP score in CGL, corroborate the interpretation of Hook and Engel. Kelemen found in cases of druginduced neutropenia and idiopathic aplastic anaemia that the NAP score was high in both peripheral blood and bone-marrow. He suggested that when the reserve of mature granulocytes in the marrow was reduced, high NAP scores were attributable to the release of newly formed neutrophils into the circulation. When marrow granulocytes were increased, as in CGL, older neutrophils which had been stored for some time and had lost their NAP activity constituted the major element in the neutrophil population of the peripheral blood. This hypothesis is compatible with the return of NAP activity in CGL under conditions where the granulocyte reserves are reduced-for example by treatment with busulphan, during the neutrophil response to infection (Rosen and Teplitz, 1965; Finney, McDonald, Baikie, and Douglas, 1972), or during the development of acute transformation, when the bone marrow is being replaced by undifferentiated leukaemic cells. We have observed an analogous phenomenon in patients without leukaemia who had extensive bone-marrow invasion by lymphoma or carcinoma: a leucoerythroblastic peripheral blood picture with neutrophilia was often accompanied by elevation of the NAP score to over 300 . The concept of reduced reserves of neutrophils with release of newly formed 
cells which possess NAP also explains the high NAP scores we observed in our CGL patients when significant bone-marrow depression had been produced by treatment with thioguanine (fig 1).

In untreated patients with CGL the enormously expanded mass of mature and immature granulocytic cells is accommodated in the bone marrow, peripheral blood, spleen, and in other extramedullary sites such as the liver and the pulmonary vasculature. If after their formation neutrophils progress from the bone-marrow multiplier compartment to storage pools, including those in the marrow itself (fig 5a) and finally to the peripheral blood, the great expansion of the storage pools in CGL should entail a delay in reaching the circulation as a definitive circulating cell. (Obviously those neutrophils which are made in the bone marrow and then stored in extramedullary pools must, if only transiently, pass through the circulation to reach their storage site.) In addition to the longer delay before becoming circulating cells, many of the neutrophils then remain for an abnormally long span in the blood (Galbraith, 1966). Thus the circulating neutrophils will be predominantly elderly and have reduced NAP activity. Reduction of the total granulocytic pool by antileukaemic drugs allows the earlier release into the circulation of younger neutrophils which possess NAP activity, and also reduces the numbers of ageing neutrophils without NAP activity, so that they no longer dilute the NAP score.

When splenectomy is performed in patients with

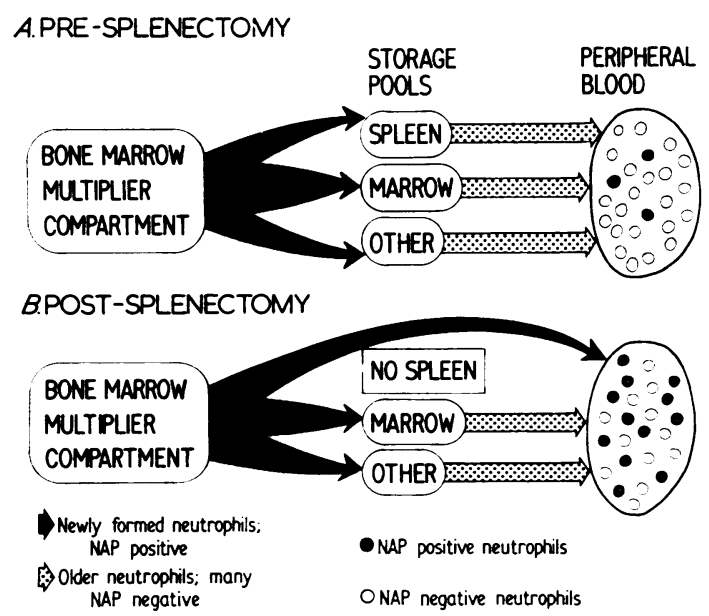

Fig 5 Postulated mechanism for the elevation of NAP score seen after splenectomy: (a) the usual situation in both normal subjects and patients with $C G L$;

(b) splenectomy permits the early circulation of newly formed neutrophils without prior storage.
CGL, an important extramedullary site for storage of neutrophils is removed (fig 5b). A temporary deficiency of storage space is reflected by a striking though transient neutrophilia, accompanied by an equally striking rise in the NAP score, which then declines in parallel with the peripheral blood neutrophil count. The rapid though usually incomplete return of both neutrophil count and NAP score to their pre-splenectomy levels (fig 4) suggests increased utilization of other storage compartments by way of compensation.

The hypothesis that NAP activity is inversely related to the age of the neutrophil conflicts with some others previously advanced. Pedersen and Hayhoe (1971b) demonstrated that actively phagocytic CGL neutrophils are more frequently NAPpositive than non-phagocytic neutrophils. This observation and evidence of defective cytoplasmic 을 maturation of many neutrophils in CGL (Pedersen and Hayhoe, 1971a) led them to postulate that the low NAP score was a manifestation of cellular immaturity. However, NAP activity has been observed in band forms (Wachstein, 1946), in oे immature granulocytic cells (Stewart, 1974), in pseudo-Pelger neutrophils (Malasková and Fuksová, 1968), and also in true Pelger neutrophils (Baikie et $a l, 1963)$. Thus immaturity or abnormal maturation do not necessarily preclude the possession of NAP by granulocytic cells. Pedersen and Hayhoe further suggested (1971b) that the principal site of origin of mature, phagocytic, NAP-positive neutrophils in CGL patients might be the spleen: an increased NAP score during acute transformation of CGL was attributed to a relatively higher proportion of the peripheral blood neutrophils being of splenic, rather than bone-marrow, origin. Our finding that splenectomy in CGL is consistently followed by a striking increase in the NAP score makes this hypothesis improbable.

We therefore postulate that fluctuations of the peripheral blood NAP score in CGL are due not to any intrinsic property peculiar to CGL cells, but to alterations in the size of the granulocyte storage compartments in the bone marrow, spleen, and elsewhere. The variable extent to which these compartments are affected by the disease and its treatment adequately explains the variations in NAP score seen in different patients whose disease appears on the basis of peripheral blood counts to have been controlled to the same extent. This hypothesis is strengthened by the finding of similar changes in NAP score when splenectomy is performed in patients who do not have leukaemia.

It may be better to express NAP activity in CGL not as a weighted score per 100 cells, but by some parameter which reflects change in the absolute 
number of mature granulocytes in the peripheral blood which have no NAP activity. Unfortunately, the current mode of expression as a weighted score per 100 cells is well established and for a variety of reasons may be difficult to replace.

We are grateful to Dr D. A. G. Galton, Honorary Director of the Medical Research Council Leukaemia Unit, and to Dr J. M. Goldman for their valued advice and criticism, and to Dr R. $M$. Lowenthal, Leukaemia Research Fund Fellow, for material from the patient treated by leucapheresis.

\section{References}

Alter, A. A., Dobkin, G., Pourfar, M., Rosner, F., and Lee, S. L. (1963). Genes on the mongol chromosome. Lancet, 1, 506. (Letter.)

Baikie, A. G. (1968). The place of splenectomy in the treatment of chronic granulocytic leukaemia: some random observations, a review of the earlier literature and a plea for its proper study in a cooperative therapeutic trial. (Abstr.) Aust. Ann. Med., 17, 175-176.

Baikie, A. G., Gillis, E. M., and King, M. J. (1963). Polymorph alkaline phosphatase activity and the Pelger-Huët anomaly. (Letter.) Lancet, 1, 670.

Bennett, J. M., Nathanson, L., and Rutenburg, A. M. (1968). Significance of leukocyte alkaline phosphatase in Hodgkin's disease. Arch. intern. Med., 121, 338-341.

Block, J. B., Carbone, P. P., Oppenheim, J. J., and Frei, E., III. (1963). The effect of treatment in patients with chronic myelogenous leukemia: biochemical studies. $A n n$. intern. Med., 59, 629-636.

Bottomley, R. H., Lovig, C. A., Holt, R., and Griffin, M. J. (1969). Comparison of alkaline phosphatase from human normal and leukemic leukocytes. Cancer Res., 29, 18661874.

Chikkappa, G., Boecker, W. R., Borner, G., Carsten, A. L., Conkling, K., Cook, L., Cronkite, E. P., and Dunwoody, S. (1973). Return of alkaline phosphatase in chronic myelocytic leukemia cells in diffusion chamber cultures. Proc. Soc. exp. Biol. (N. Y.), 143, 212-217.

Finney, R., McDonald, G. A., Baikie, A. G., and Douglas, A. S. (1972). Chronic granulocytic leukaemia with $\mathbf{P h}^{\mathbf{1}}$ negative cells in bone marrow and a ten year remission after busulphan hypoplasia. Brit. J. Haemat., 23, 283-288.

Galbraith, P. R. (1966). Studies on the longevity, sequestration and release of the leukocytes in chronic myelogenous leukemia. Canad. med. Ass. J., 95, 511-521.

Hammouda, F., Quaglino, D., and Hayhoe, F. G. J. (1964). Blastic crisis in chronic granulocytic leukaemia. Cytochemical cytogenetic, and autoradiographic studies in four cases. Brit. med. J., 1, 1275-1281.

Hook, E. B. and Engel, R. R. (1964). Leucocyte life-span, leucocyte alkaline phosphatase, and the 21 st chromosome. (Letter.) Lancet, 1, 112.

Kelemen, E. (1973). Granulocyte alkaline phosphatase activity: a measure of the emergence time of mature marrow neutrophils? Acta Haemat. (Basel), 50, 19-24.

King, M. J., Gillis, E. M., and Baikie, A. G. (1962). Alkalinephosphatase activity of polymorphs in mongolism. Lancet, 2, 1302-1305.

Koler, R. D., Seaman, A. J., Osgood, E. E., and Van-
Bellinghen, P. (1958). Myeloproliferative diseases: diagnostic value of the leukocyte alkaline phosphatase test. Amer. J. clin. Path., 30, 295-301.

Lewis, S. M. and Dacie, J. V. (1965). Neutrophil (leucocyte) alkaline phosphatase in paroxysmal nocturnal haemoglobinuria. Brit. J. Haemat., 11, 549-556.

Lille-Szyszkowicz, I., Gabay, P., Saracino, R. T., and Bourdin, J. S. (1966). La phosphatase alcaline leucocytaire chez les malades atteints de tumeurs ou d'hémopathies malignes. Nouv. Rev. franc. Hémat., 6, 187-194.

Malasková, V. and Fuksová, J. (1968). Alkaline phosphatase activity in immature granulocytes. Brit. J. Haemat., 15, 119-122.

Mitus, W. J., Bergna, L. J., Mednicoff, I. B., and Dameshek, W. (1958). Alkaline phosphatase of mature neutrophils in chronic forms of the myeloproliferative syndrome. Amer. J. clin. Path., 30, 285-294.

O'Riordan, M. L., Robinson, J. A., Buckton, K. E., and Evans, H. J. (1971). Distinguishing between the chromosomes involved in Down's syndrome (trisomy 21) and chronic myeloid leukaemia $\left(\mathrm{Ph}^{1}\right)$ by fluorescence. Nature (Lond.), 230, 167-168.

Pedersen, B. and Hayhoe, F. G. J. (1971a). Cellular changes in chronic myeloid leukaemia. Brit.J. Haemat., 21, 251-256.

Pedersen, B. and Hayhoe, F. G. J. (1971b). Relation between phagocytic activity and alkaline phosphatase content of neutrophils in chronic myeloid leukaemia. Brit.J. Haemat., 21, 257-260.

Rosen, R. B. and Teplitz, R. L. (1965). Chronic granulocytic leukemia complicated by ulcerative colitis: elevated leukocyte alkaline phosphatase and possible modifier gene deletion. Blood, 26, 148-156.

Rosner, F., Schreiber, Z. R., and Parise, F. (1972). Leukocyte alkaline phosphatase: fluctuations with disease status in chronic granulocytic leukemia. Arch. intern. Med., 130, 892-894.

Rutenburg, A. M., Rosales, C. L., and Bennett. J. M. (1965). An improved histochemical method for the demonstration of leukocyte alkaline phosphatase activity: clinical application. J. Lab. clin. Med., 65, 698-705.

Sforza, M. B., De Somoza, N., and Morgenfeld, C. M. (1971). Significado de la fosfatasa alcalina leucocitaria en la enfermedad de Hodgkin. Sangre, 16, 411-416.

Simmons, A. V., Spiers, A. S. D., and Fayers, P. M. (1973). Haematological and clinical parameters in assessing activity in Hodgkin's disease and other malignant lymphomas. Quart. J. Med., 42, 111-124.

Spiers, A. S. D. (1973). Surgery in management of patients with leukaemia. Brit. med. J., 3, 528-532.

Stewart, C. A. (1974). Leucocyte alkaline phosphatase in myeloid maturation. Pathology, 6, 287-293.

Strausz, I., Kékes, E., and Szebeni, A. (1965). Mechanism of prednisolone-induced leukocytosis in man. Acta haemat. (Basel), 33, 40-48.

Tanaka, K. R., Valentine, W. N., and Fredricks, R. E. (1960). Diseases or clinical conditions associated with a low leukocyte alkaline phosphatase. New Engl. J. Med., 262, 912-918.

Tough, I. M., Jacobs, P. A., Court Brown, W. M., Baikie, A. G., and Williamson, E. R. D. (1963). Cytogenetic studies on bone marrow in chronic myeloid leukaemia. Lancet, 1, 844-846.

Wachstein, M. (1946). Alkaline phosphatase activity in normal and abnormal human blood and bone marrow cells. J. Lab. clin. Med., 31, 1-17. 